\title{
AICAR induces mitochondrial apoptosis in human osteosarcoma cells through an AMPK-dependent pathway
}

\author{
MASAYUKI MORISHITA $^{1}$, TERUYA KAWAMOTO ${ }^{1}$, HITOMI HARA ${ }^{1}$, YASUO ONISHI $^{1}$, TAKESHI UEHA ${ }^{2}$, \\ MASAYA MINODA ${ }^{1}$, ETSUKO KATAYAMA ${ }^{1}$, TOSHIYUKI TAKEMORI ${ }^{1}$, NAOMASA FUKASE ${ }^{1}$, \\ MASAHIRO KUROSAKA ${ }^{1}$, RYOSUKE KURODA ${ }^{1}$ and TOSHIHIRO AKISUE ${ }^{1,3}$ \\ ${ }^{1}$ Department of Orthopaedic Surgery, ${ }^{2}$ Division of Rehabilitation Medicine, \\ Kobe University Graduate School of Medicine, Kobe 650-0017; ${ }^{3}$ Department of Rehabilitation Science, \\ Kobe University Graduate School of Health Sciences, Kobe 654-0142, Japan
}

Received August 8, 2016; Accepted November 4, 2016

DOI: 10.3892/ijo.2016.3775

\begin{abstract}
The AMP-activated protein kinase (AMPK) activator 5-aminoimidazole-4-carboxamide ribonucleotide (AICAR) modulates cellular energy metabolism, and promotes mitochondrial proliferation and apoptosis. Previous studies have shown that AICAR has anticancer effects in various cancers, however the roles of AMPK and/or the effects of AICAR on osteosarcoma have not been reported. In the present study, we evaluated the effects of AICAR on tumor growth and mitochondrial apoptosis in human osteosarcoma both in vitro and in vivo. For in vitro experiments, two human osteosarcoma cell lines, MG63 and KHOS, were treated with AICAR, and the effects of AICAR on cell growth and mitochondrial apoptosis were assessed by WST assays, TUNEL staining, and immunoblot analyses. In vivo, human osteosarcoma-bearing mice were treated with AICAR, and the mitochondrial proliferation and apoptotic activity in treated tumors were assessed. In vitro experiments revealed that AICAR activated AMPK, inhibited cell growth, and induced mitochondrial apoptosis in both osteosarcoma cell lines. In vivo, AICAR significantly reduced osteosarcoma growth without apparent body weight loss and AICAR increased both mitochondrial proliferation and apoptotic activity in treated tumor tissues. AICAR showed anticancer effects in osteosarcoma cells through an AMPK-dependent peroxisome proliferator-activated receptor- $\gamma$ coactivator- $1 \alpha$ (PGC-1 $\alpha$ )/mitochondrial transcription factor A (TFAM)/mitochondrial pathway. The findings in this study strongly suggest
\end{abstract}

Correspondence to: Dr Teruya Kawamoto, Department of Orthopaedic Surgery, Kobe University Graduate School of Medicine, 7-5-1 Kusunoki-cho, Chuo-ku, Kobe 650-0017, Japan

E-mail: trykwmt@med.kobe-u.ac.jp

Key words: osteosarcoma, AMP-activated protein kinase, 5-aminoimidazole-4-carboxiamide ribonucleotide, mitochondria, peroxisome proliferator-activated receptor- $\gamma$ coactivator- $1 \alpha$, mitochondrial transcription factor A that AICAR could be considered as a potent therapeutic agent for the treatment of human osteosarcoma.

\section{Introduction}

Osteosarcoma is the most common primary malignant bone tumor in adolescents and young adults. Currently, the most effective therapeutic option for the osteosarcoma is surgical resection of all clinically detectable sites with systemic therapy to control microscopic metastatic disease. Many studies have reported significant advances in adjuvant chemotherapy for osteosarcoma $(1,2)$, however, in the last two decades, treatment outcomes for patients with osteosarcoma have not improved sufficiently, despite the implementation of several new therapeutic interventions. In a comprehensive analysis of published phase I/II clinical trials, researchers found that effective drugs tended to have high toxicity (3). Therefore, new therapeutic strategies for the treatment of osteosarcoma are needed.

Mitochondria are cytoplasmic organelles that play essential roles in cellular energy metabolism and programmed cell death (4), and regulation of both mitochondrial dysfunction and associated cellular biogenetics has recently been identified as a promising target for anticancer therapy (5). Moreover, quantitative changes in mitochondrial numbers have been observed in various cancers, with a decrease in hepatocellular carcinoma, renal cell carcinoma, advanced gastric cancer and breast cancer (6-11) and with an increase in head and neck cancers, ovarian cancer, and esophageal squamous cell carcinoma (12-14). Additionally, decreased mitochondrial numbers have been reported to be associated with tumor progression and prognosis in patients with hepatocellular carcinoma and breast cancer $(6,7)$. We previously reported that mitochondrial numbers were significantly reduced in human sarcoma tissues, including osteosarcoma, compared with that in normal muscles or benign musculoskeletal tumor tissues, and that decreased mitochondrial numbers may be associated with tumor progression in musculoskeletal malignancies (15). In mitochondrial biogenesis, peroxisome proliferator-activated receptor- $\gamma$ coactivator- $1 \alpha$ (PGC- $1 \alpha$ ) regulates the transcription of the gene coding mitochondrial transcription factor A (TFAM), which reflects the changing 
levels of mitochondrial DNA (mtDNA) in the cell, and plays a crucial role in mtDNA maintenance (16). We have recently reported that increasing mitochondrial numbers by forced expression of PGC- $1 \alpha$ could induce mitochondrial apoptosis in human sarcoma cell lines (15), and the findings suggested that mitochondrial biogenesis and/or numbers could be a therapeutic target for human sarcomas. 5-Aminoimidazole4-carboxiamide ribonucleotide (AICAR) is widely used as a pharmacologic activator of the energy-regulating enzyme AMP-activated protein kinase $\alpha(\mathrm{AMPK} \alpha)(17,18)$. AICAR has been also reported to stimulate mitochondrial biogenesis via PGC-1 $\alpha$ activation in skeletal muscles (19) and to be an exercise mimetic (20).

Based on these previous studies, we hypothesized that AICAR could increase PGC-1 $\alpha$ expression through AMPK activation and could induce mitochondrial apoptosis through the PGC-1 $\alpha /$ TFAM/mitochondrial pathway in tumor tissues similarly to muscle tissues. Moreover, we expected that AICAR may show anticancer effects in osteosarcoma cells. Therefore, in this study, we evaluated the effects of AICAR on apoptotic activity through AMPK phosphorylation in human osteosarcoma cells both in vitro and in vivo.

\section{Materials and methods}

Reagents. AICAR was obtained from Abcam (Cambridge, UK) (ab120358, Lot: APN12423-1-1; ) for cell experiments, and LC Laboratories (Woburn, MA, USA) (A-1098, Lot: ACR-101) for animal experiments. AICAR was dissolved in distilled water and immediately stored at $-20^{\circ} \mathrm{C}$. This stock solution was diluted in culture medium for in vitro or saline for in vivo experiments, immediately before use.

Cells. Two human osteosarcoma cell lines (MG63 and KHOS) were used in this study. MG63 cells were obtained from the RIKEN BRC through the National Bio-Resource Project of the MEXT (Ibaraki, Japan), and KHOS cells were obtained from American Type Culture Collection (ATCC, Manassas, VA, USA). Both cell lines were routinely cultured in Dulbecco's modified Eagle's medium (DMEM) containing 10\% fetal bovine serum (FBS) and $100 \mathrm{U} / \mathrm{ml}$ penicillin/streptomycin solution (all from Sigma-Aldrich, St. Louis, MO, USA) at $37^{\circ} \mathrm{C}$ in a humidified $5 \% \mathrm{CO}_{2}$ atmosphere. For all experiments, we used DMEM containing $10 \%$ FBS without the antibiotic solution.

Animal studies. All animal experiments were approved by Kobe University Animal Experimentation Regulations (permission no. P-140504). Male BALB/c nude mice, aged 5 weeks, were purchased from CLEA Japan Inc. (Tokyo, Japan) and were maintained in a facility under specific pathogen-free conditions. Animals were fed pathogen-free laboratory chow and allowed free access to autoclaved water in an air-conditioned room with a 12-h light/dark cycle. For in vivo experiments, both MG63 and KHOS osteosarcoma cells were implanted into the dorsal, subcutaneous area of mice $(n=12$ for each cell line) at a dose of $2.0 \times 10^{6}$ cells in $500 \mu$ phosphate-buffered saline (PBS), as previously described (21), and mice were randomly divided into two groups: the AICAR-treated group ( $n=6$ for each cell line) and the control group $(n=6$ for each cell line). Treatment with $450 \mathrm{mg} / \mathrm{kg}$ AICAR or saline (as a control) by intraperitoneal injection was started after 1 week of cell implantation and was performed every day for 2 weeks. Tumor volume was calculated twice a week, as previously described, according to the formula $V=\pi / 6 \mathrm{xa}^{2} \mathrm{xb}$, where $a$ and $b$ represent the shorter and longer dimensions of the tumor, respectively (21). The body weight of each mouse was also monitored throughout the experiment. At the end of the experiment, all tumors were excised and immediately stored at $-80^{\circ} \mathrm{C}$, and mitochondrial proliferation and apoptotic activity in tumor tissues were evaluated by flow cytometry, immunoblotting, and immunofluorescence staining.

Immunoblot analysis. Cell lysates were extracted from cells or implanted tumors using whole cell lysis buffer (Mammalian Protein Extraction reagent; Thermo Scientific, Rockford, IL, USA) and protein content was quantified using BCA protein assay reagent (Bio-Rad, Hercules, CA, USA). Samples containing equal amounts of protein were electrophoresed by sodium dodecyl sulphate polyacrylamide gel electrophoresis (SDS-PAGE) on 7.5-15\% gradient gels and transferred to polyvinylidene fluoride membranes. After blocking, membranes were incubated overnight at $4^{\circ} \mathrm{C}$ with primary antibodies in CanGet Signal Solution 1 (Toyobo Co., Ltd., Osaka, Japan). The following primary antibodies were used in this study: anti-human phospho-AMPK $\alpha$ (Thr172) antibody (1:1,000) (2531S, Lot: 12), anti-human AMPK $\alpha$ antibody $(1: 1,000)(2532 S, L o t: 19)$ (both from Cell Signaling Technology, Danvers, MA, USA), anti-human PGC-1 $\alpha$ antibody $(1: 1,000)$ (H000110891-M12, Lot: 08162-3G11), anti-human TFAM antibody $(1: 1,000)$ (H00007019-B01P, Lot: E1281) (both from Abnova, Walnut, CA, USA), anti-human poly(ADP-ribose) polymerase (PARP) antibody (1:1,000) (9542S, Lot: 13), anti-human cleaved PARP antibody (1:1,000) (5625S, Lot: 8), anti-human caspase-3 antibody (1:1,000) (9668S, Lot: 7), antihuman cleaved caspase-3 antibody $(1: 1,000)$ (9664S, Lot: 20), anti-human caspase-9 antibody (1:1,000) (9502S, Lot:8), antihuman cleaved caspase-9 antibody (1:500) (7237S, Lot: 1) (all from Cell Signaling Technology), and anti-human $\alpha$-tubulin antibody (1:10,000) (T9026, Lot: 092M4792; Sigma-Aldrich). After washing, membranes were incubated with the appropriate secondary antibody conjugated to horseradish peroxidase and were exposed with ECL Plus Western Blot Detection system reagents (GE Healthcare Biosciences, Piscataway, NJ, USA), and protein expression was detected using a Chemilumino Analyzer LAS-3000 mini (Fujifilm, Tokyo, Japan). Membranes were reprobed with anti-human $\alpha$-tubulin antibody (SigmaAldrich) to confirm equal protein loading. Positive bands in immunoblot analyses were semiquantified by densitometrical analyses using the Image J software (version 1.47; NIH, Bethesda, MD, USA) (http://rsbweb.nih.gov/ij/download.html). Values were normalized against $\alpha$-tubulin and presented as a ratio.

Quantitative real-time polymerase chain reaction ( $q P C R)$. To evaluate mitochondrial numbers in AICAR-treated osteosarcoma cell lines, we examined the relative amount of mtDNA to nuclear DNA (nDNA). Genomic DNA was isolated from the cells using a GenElute Mammalian Genomic DNA Miniprep kit (Sigma-Aldrich). The sequences of the primers designed to amplify a region corresponding to nn 16-408 of a D-loop of 
human mtDNA were as follows: 5'-GCAGATTTGGGTACCAC CCAAGTATTGACTCACCC-3' (forward) and 5'-GCATGGAG AGCTCCCGTGAGTGGTTAATAGGGTGATAG-3' (reverse). The PCR conditions were as follows: 1 cycle at $95^{\circ} \mathrm{C}$ for $15 \mathrm{~min}$, followed by 30 cycles at $95^{\circ} \mathrm{C}$ for $30 \mathrm{sec}, 58^{\circ} \mathrm{C}$ for $30 \mathrm{sec}$, and $72^{\circ} \mathrm{C}$ for $90 \mathrm{sec}(22)$. The relative amount of mtDNA to nDNA was then analysed using the $\Delta \Delta \mathrm{Ct}$ method.

Cell proliferation assays. To evaluate the effects of AICAR on in vitro osteosarcoma cell proliferation, we performed WST-8 cell proliferation assays using a cell counting kit-8 (CCK-8; Dojindo Inc., Kumamoto, Japan). Cells were seeded in 96-well culture plates at a density of 5,000 cells/well in $100 \mu \mathrm{l}$ medium and incubated with various concentrations of AICAR $(0-2,000 \mu \mathrm{M})$. At the indicated incubation times, $10 \mu \mathrm{l}$ of the CCK-8 solution was added into each well and incubated for $1 \mathrm{~h}$. Then, the optical density was measured at a wavelength of $450 \mathrm{~nm}$ using a Model 680 Microplate Reader (Bio-Rad), and the relative number of viable cells in each well was calculated.

Immunofluorescence staining. Immunofluorescence staining was performed to verify the relationship between mitochondrial proliferation and cellular apoptosis in AICAR-treated osteosarcoma cells and implanted tumors using an APO-Direct kit (BD Pharmingen, Franklin Lakes, NJ, USA) and a MitoTracker Deep-Red FM (Invitrogen, Carlsbad, CA, USA) according to the manufacturers' protocols. In vitro, cells were incubated with or without AICAR and then fixed in $4 \%$ paraformaldehyde for $30 \mathrm{~min}$ at room temperature. Cells were incubated in the prepared DNA Labelling Solution (APO-Direct kit; BD Pharmingen) for $1 \mathrm{~h}$, and were then incubated in the MitoTracker Deep-Red FM (Invitrogen) for $30 \mathrm{~min}$. Nuclear staining was performed using a propidium iodide, and stained cells were assessed using a BZ-8000 confocal microscope (Keyence, Osaka, Japan). In vivo, tumor tissue samples were embedded in an OCT compound (Sakura Finetek Co., Tokyo, Japan), and 10- $\mu$ m-thick sections were prepared on a cryostat and stored frozen at $-80^{\circ} \mathrm{C}$. Sections were incubated with anti-actin antibodies (Sigma-Aldrich) diluted in PBS for $30 \mathrm{~min}$ at $37^{\circ} \mathrm{C}$. After washing, sections were incubated with the APO-Direct kit reagents (BD Pharmingen) and the MitoTracker Deep-Red FM (Invitrogen) in PBS for 30 min in a dark humid chamber at $37^{\circ} \mathrm{C}$. The nucleus was stained with the 4',6-diamidino-2-phenylindole (DAPI). Fluorescence images were obtained using a BZ-8000 confocal microscope (Keyence), and positive staining was semiquantified using ImageJ software.

Flow cytometric analysis. We also assessed apoptotic activity in AICAR-treated cells or implanted tumors by flow cytometry. Briefly, cells were collected from cultured cells or implanted tumors, suspended in $1 \%$ paraformaldehyde in PBS, and resuspended in ice-cold ethanol at a concentration of $1 \times 10^{6}$ cells $/ \mathrm{ml}$. Each cell pellet was labelled using the APO-Direct kit (BD Pharmingen) according to the manufacturers' protocols. Fluorescent intensity was analysed using a BD FACSVerse (BD Biosciences, Franklin Lakes, NJ, USA).

Statistical analysis. Each experiment was performed independently at least three times. All values are expressed as the mean \pm standard error of the mean (SEM). Comparisons between two continuous values were made using one-way analysis of variance. Post-hoc analysis was performed by Fisher's protected least significant difference test. For distributed data, p-values $<0.05$ were considered significant.

\section{Results}

AICAR increases AMPK phosphorylation and induces mito-

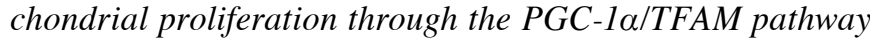
in osteosarcoma cells. We first examined the effects of AICAR on AMPK phosphorylation and mitochondrial biogenesis in osteosarcoma cells in vitro. As shown in Fig. 1, the expression of phosphorylated AMPK $\alpha$ (Thr172) was strongly increased immediately after AICAR treatment in both MG63 and KHOS osteosarcoma cells (Fig. 1A and B). Additionally, AICAR significantly increased the relative number of mtDNA copies in both cell lines (Fig. 1C). Consistent with these results, the expression levels of the mitochondria-related genes, PGC-1 $\alpha$ and TFAM, were increased in AICAR-treated osteosarcoma cells compared with those in control cells (Fig. 1D). These findings strongly suggested that AICAR could induce AMPK activation and increase mitochondrial biogenesis through the PGC-1 $\alpha /$ TFAM pathway in osteosarcoma cells.

AICAR decreases osteosarcoma cell growth and induces mitochondrial apoptosis and proliferation. To test the effects of mitochondrial proliferation by AICAR on osteosarcoma cell growth and apoptosis, we assessed cell viability and apoptotic activity in osteosarcoma cells after AICAR treatment. AICAR showed concentration-dependent inhibitory effects on cell viability in both osteosarcoma cell lines (Fig. 2A). Flow cytometric analyses revealed that AICAR strongly increased the number of apoptotic cells (Fig. 2B), and immunoblot analyses demonstrated that cleaved forms of caspase-9, caspase- 3 and PARP were strongly increased in osteosarcoma cells after $72 \mathrm{~h}$ of treatment with 1,000 $\mu \mathrm{M}$ AICAR (Fig. 2C). Additionally, immunofluorescence staining revealed that AICAR treatment increased the apoptotic cell numbers along with increased mitochondrial proliferation in both osteosarcoma cells (Fig. 3). Semi-quantitative analyses of immunofluorescence positive staining showed that apoptotic cells significantly increased in AICAR-treated cells, and the relative positive staining to control was a 6.87- and a 24.6-fold increase in MG63 and KHOS cells, respectively $(\mathrm{p}<0.05)$. Positive staining of mitochondria also significantly increased after AICAR treatment, and the relative positive staining to control was a 3.43- and a 10.7-fold increase in MG63 and KHOS cells, respectively $(p<0.05)$. These observations suggested that AICAR-dependent mitochondrial proliferation induced mitochondrial apoptosis in human osteosarcoma cells.

AICAR suppressed tumor growth in human osteosarcoma xenografts with increased mitochondrial apoptosis. We evaluated the in vivo antitumor activity of AICAR using human osteosarcoma xenografts. In both osteosarcoma cell implanted models, AICAR significantly suppressed in vivo osteosarcoma tumor growth compared with the growth of control tumors (Fig. 4). At the end of the experiment, tumor volume in the AICAR-treated group was $85.9 \%$ in MG63 (Fig. 4A) and 
$\mathbf{A}$

MG63

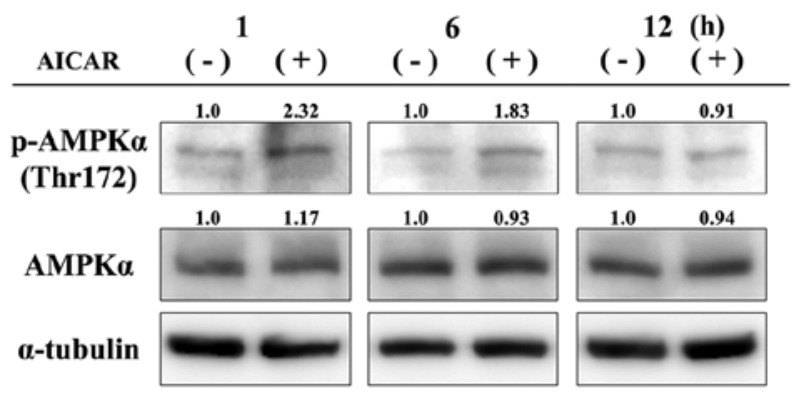

C

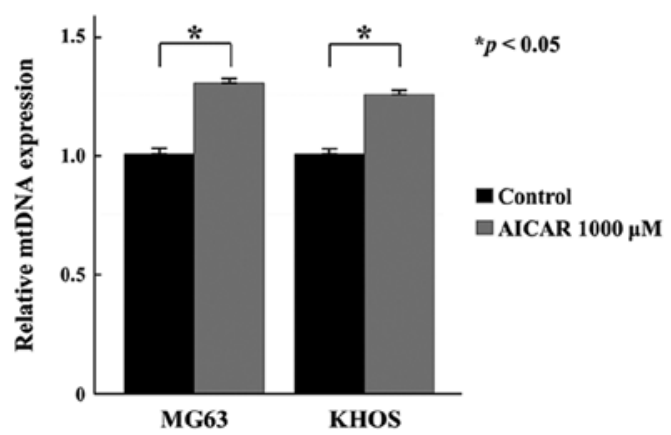

B KHOS

\begin{tabular}{|c|c|c|c|c|c|c|}
\hline \multirow[b]{2}{*}{ AICAR } & \multicolumn{2}{|c|}{1} & \multicolumn{2}{|c|}{6} & \multicolumn{2}{|c|}{12 (h) } \\
\hline & $(-)$ & $(+)$ & $(-)$ & $(+)$ & & $(+)$ \\
\hline & 1.0 & 1.43 & 1.0 & 1.95 & 1.0 & 0.96 \\
\hline & inds & 96 & twing & 50 & ther & 58 \\
\hline & 1.0 & 1.12 & 1.0 & 0.96 & 1.0 & 1.0 \\
\hline AMPK $\alpha$ & $=$ & $=4$ & $m$ & $=$ & 10 & 20 \\
\hline -tubulin & & & & & & \\
\hline
\end{tabular}

D MG63 $\underline{\text { KHOS }}$

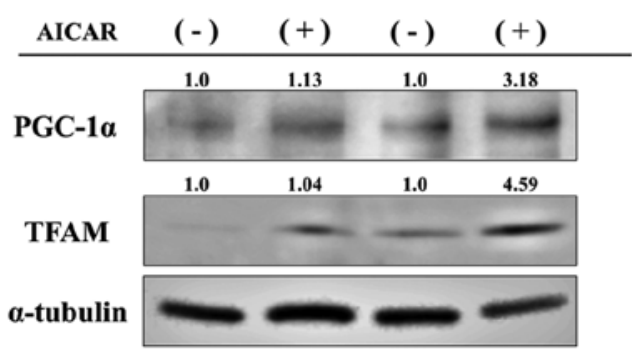

Figure 1. AMPK phosphorylation and mitochondrial proliferation after AICAR treatment in osteosarcoma cells. (A and B) The levels of AMPK $\alpha$ and phosphorylated AMPKa (Thr172) were evaluated by immunoblot analyses after 30 min, 1 and $6 \mathrm{~h}$ of AICAR treatment (1,000 $\mu$ M) in (A) MG63 and (B) KHOS osteosarcoma cells. Positive bands were semiquantified by densitometrical analyses using ImageJ software. Values were normalized against $\alpha$-tubulin and presented as a ratio. (C) Relative mtDNA copy numbers to nDNA in AICAR-treated osteosarcoma cell lines were evaluated by quantitative real-time PCR after $72 \mathrm{~h}$ of treatment. Data represent the mean \pm SEM of at least three independent experiments ("p $<0.05)$. (D) Immunoblot analysis of PGC-1 $\alpha$ and TFAM in AICAR-treated osteosarcoma cell lines. Positive bands were semiquantified by densitometrical analyses using ImageJ software. Values were normalized against $\alpha$-tubulin and presented as a ratio.

A

$\underline{\text { MG63 }}$

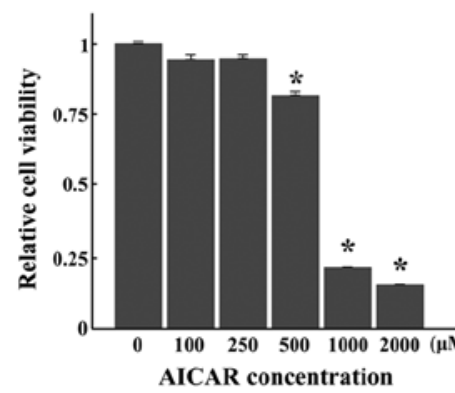

B

$\underline{\text { MG63 }}$

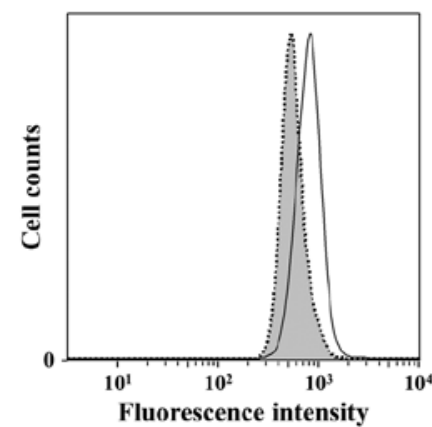

- Contro
KHOS

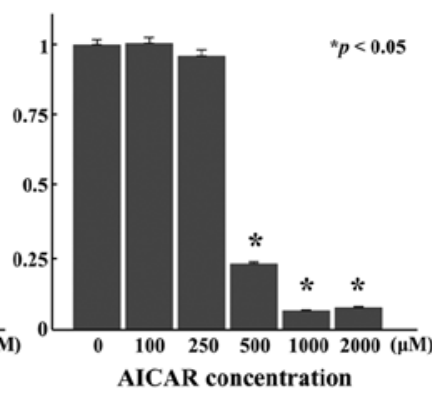

KHOS

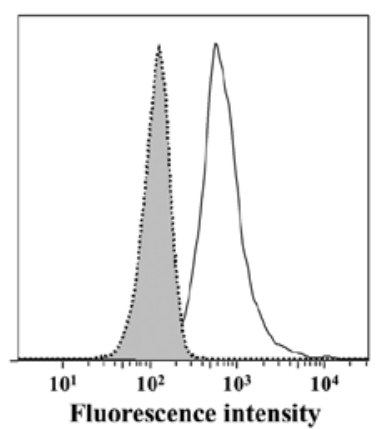

C

MG63

KHOS

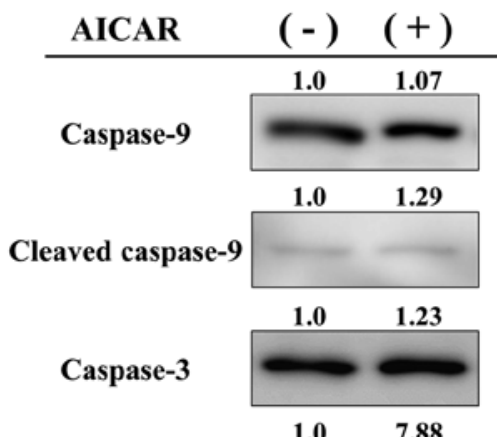

$(-) \quad(+)$

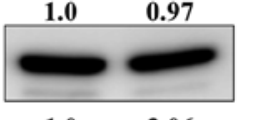

$1.0 \quad 3.06$

Cleaved caspase-3
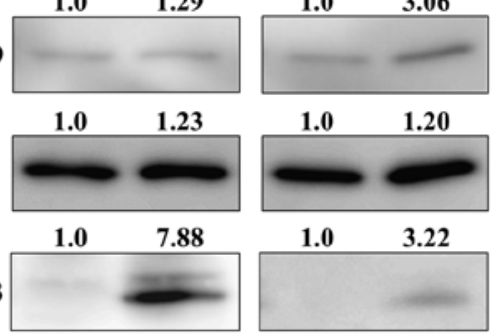

$1.0 \quad 3.22$
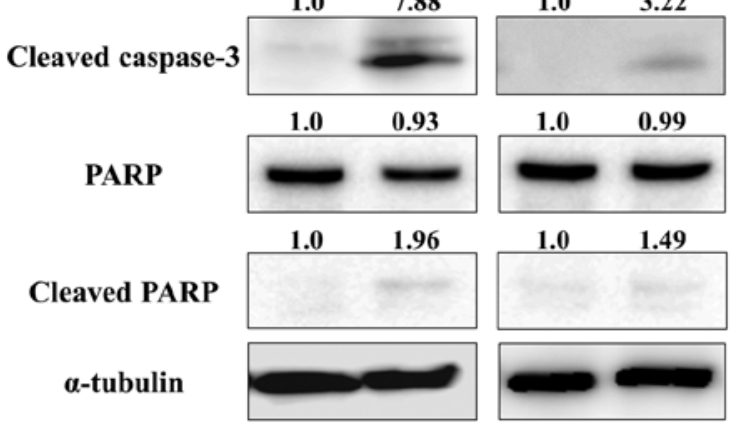

Figure 2. Effects of AICAR on cell viability and apoptotic activity in human osteosarcoma cell lines. (A) Relative cell viability was assessed by WST-8 assays in AICAR-treated human osteosarcoma cell lines after $72 \mathrm{~h}$ of treatment. Data represent the mean \pm SEM of at least three independent experiments ("p $<0.05$ ). (B) DNA fragmentation was assessed by flow cytometry after $72 \mathrm{~h}$ of AICAR treatment $(1,000 \mu \mathrm{M})$. (C) Immunoblot analysis of the apoptosis-related proteins caspase-9, caspase-3 and PARP and their cleaved forms in AICAR-treated osteosarcoma cell lines. Positive bands were semiquantified by densitometrical analyses using ImageJ software. Values were normalized against $\alpha$-tubulin and presented as a ratio. 
$\mathbf{A}$

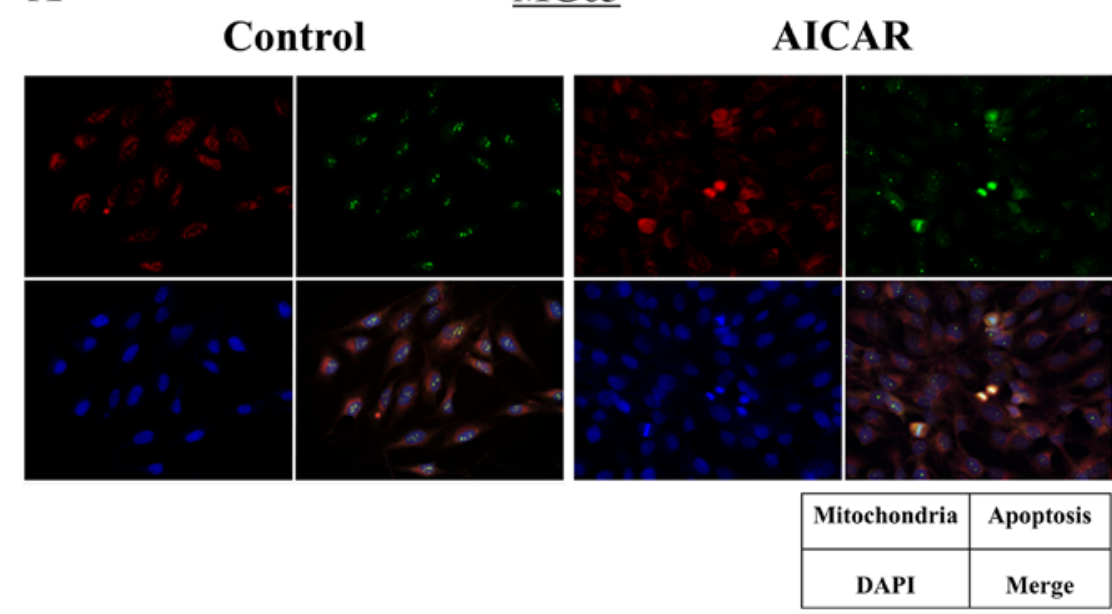

B

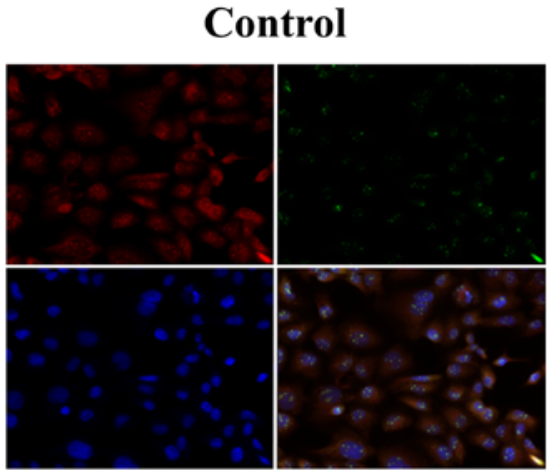

$\underline{\text { KHOS }}$

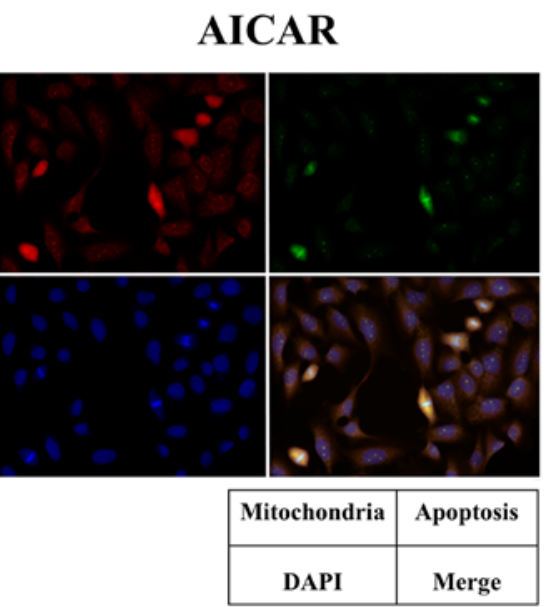

Figure 3. Immunofluorescence staining for mitochondria and apoptotic cells in AICAR-treated osteosarcoma cell lines. Mitochondrial proliferation and apoptotic activity after AICAR treatment $(1,000 \mu \mathrm{M})$ were evaluated by immunofluorescence staining in both (A) MG63 and (B) KHOS osteosarcoma cell lines. Red, mitochondria (MitoTracker Deep-Red FM); green, apoptotic nuclei (APO-Direct); blue, nuclei (DAPI).

$64.6 \%$ in KHOS (Fig. 4B) of the volume in each control group $(\mathrm{p}<0.05)$. No significant loss in body weight was observed during the experimental period in either osteosarcoma cell model (Fig. 4). In flow cytometry (Fig. 5A) and immunoblot analyses (Fig. 5B), increased apoptotic activity was observed in AICAR-treated KHOS tumor tissues, and the expression levels of the mitochondria-related genes, PGC- $1 \alpha$ and TFAM, were also increased in AICAR-treated KHOS tumors (Fig. 5C). Additionally, in immunofluorescence staining, apoptotic cells and mitochondrial proliferation were observed in AICAR-treated both osteosarcoma tumor tissues, consistent with the results from in vitro experiments (Fig. 5D and E). These findings suggested that AICAR could induce apoptosis via mitochondrial proliferation in human osteosarcoma in vivo.

\section{Discussion}

Osteosarcoma is the most common primary bone tumor in childhood and adolescence and is a clinically aggressive disease. Present therapeutic strategies, including a combination of chemotherapy and surgery, have been shown to improve a long-term survival in $\sim 60-70 \%$ of cases; however, the prognosis of patients with metastatic or recurrent disease is still poor because of the lack of second-line chemotherapies $(23,24)$. Therefore, new therapeutic strategies against high-grade osteosarcomas need to be established.

Mitochondria play essential roles in programmed cell death (4), and in mitochondrial biogenesis, the PGC-1 $\alpha /$ TFAM pathway plays a crucial role in mtDNA maintenance (16). In various human malignancies, quantitative changes in mitochondrial numbers have been observed (6-11), and decreased mitochondrial numbers have been reported to be associated with tumor progression and prognosis in patients with cancers $(6,7)$. Therefore, regulation of mitochondrial dysfunction has been identified as a promising target for anticancer therapy (5). We have previously reported that mitochondrial numbers are significantly reduced in human sarcoma tissues, including osteosarcomas, and that increasing the mitochondrial numbers by forced expression of PGC-1 $\alpha$ induces mitochondrial apoptosis in human sarcoma cell lines that exhibit decreased mitochondrial numbers (15). Thus, because these findings suggest that decreased mitochondrial numbers may influence tumor progression in malignant 
A

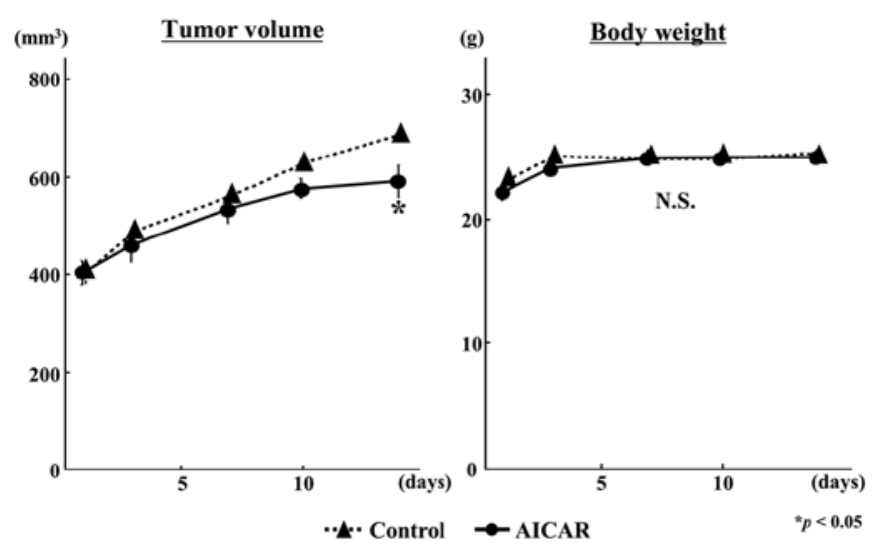

B

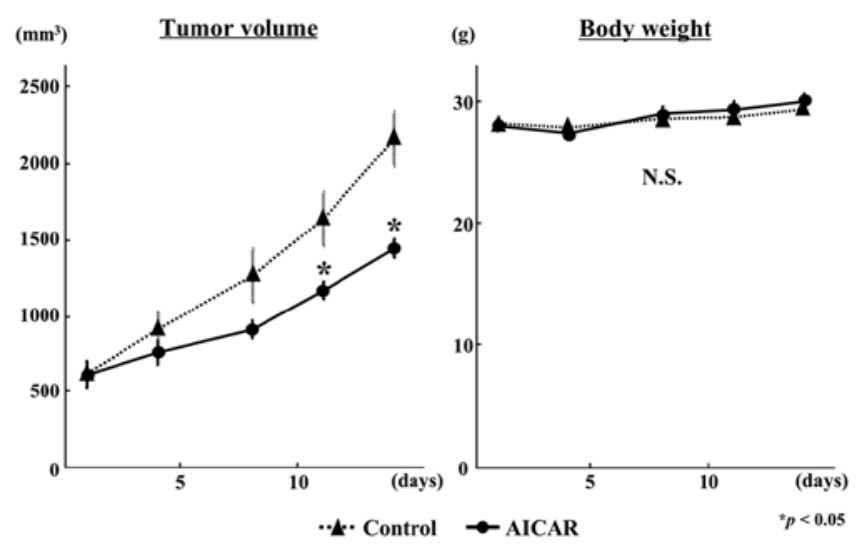

Figure 4. In vivo antitumor activities of AICAR in human osteosarcoma xenografts. Tumor volumes ( $\mathrm{mm}^{3}$ ) and body weights (g) in (A) MG63 and (B) KHOS cell-bearing mice treated with or without AICAR $(450 \mathrm{mg} / \mathrm{kg} / \mathrm{day})$ for 14 days. Data represent the mean $\pm \mathrm{SEM}$ of at least three independent experiments ("p $<0.05$ ).

A

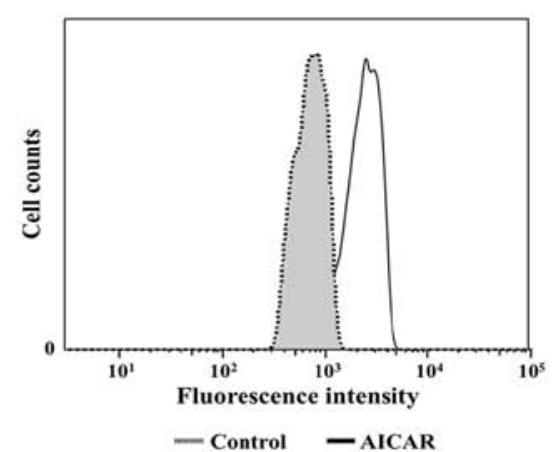

D

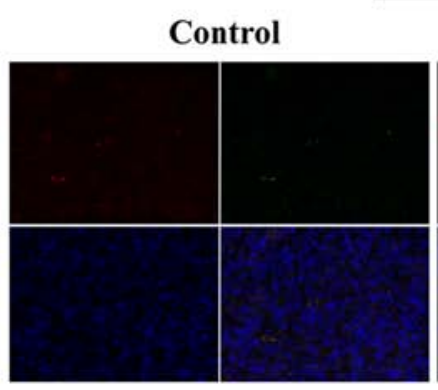

B

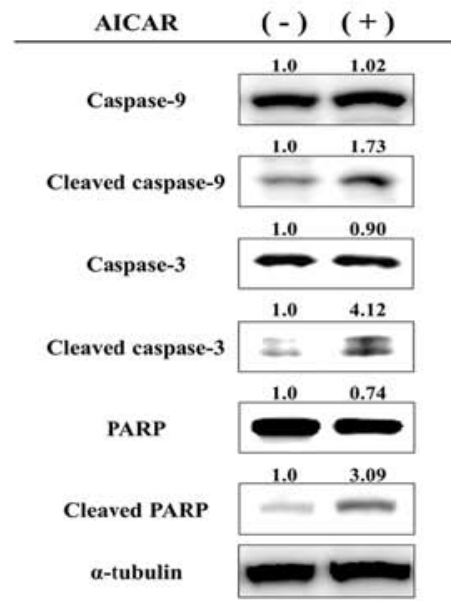

C

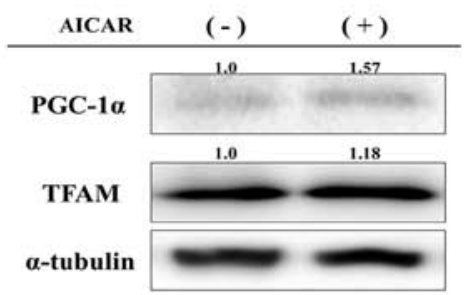

$\mathbf{E}$

AICAR

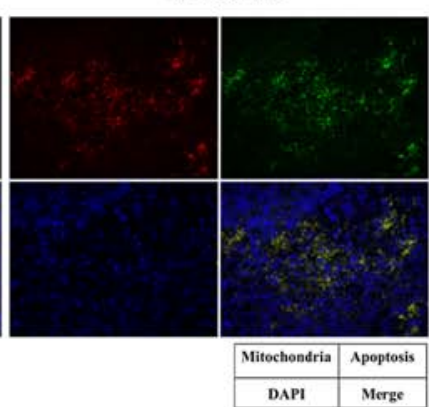

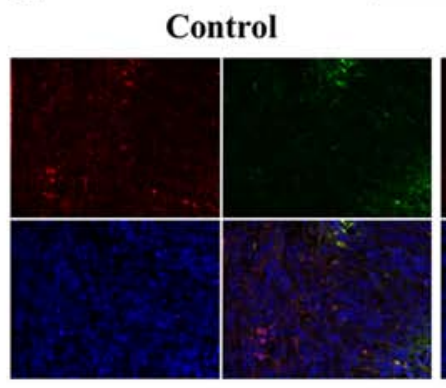

$\underline{\text { KHOS }}$

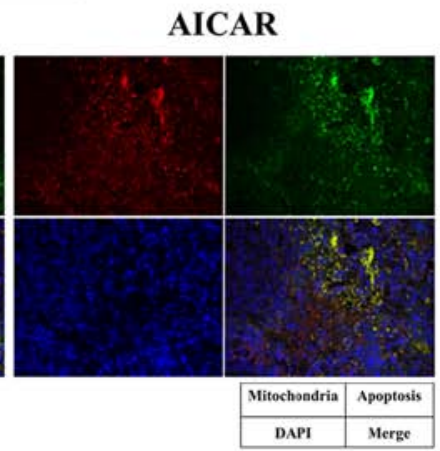

Figure 5. Effects of AICAR on mitochondrial apoptosis in human osteosarcoma xenografts. (A) DNA fragmentation in KHOS cell-derived tumor tissues after 14 days of AICAR treatment was assessed by flow cytometry. (B and C) Immunoblot analysis of (B) apoptosis-related proteins and (C) mitochondrial factors (PGC-1 $\alpha$ and TFAM) in AICAR-treated or control KHOS cell-derived tumor tissues. Positive bands were semiquantified by densitometrical analyses using the ImageJ software. Values were normalized against $\alpha$-tubulin and presented as a ratio. (D and E) Mitochondrial proliferation and DNA fragmentation in (D) MG63 and (E) KHOS cell-derived tumor tissues from AICAR-treated and control mice were evaluated by immunofluorescence staining. Red, mitochondria (MitoTracker Deep-Red FM); green, apoptotic nuclei (APO-Direct); blue, nuclei (DAPI).

musculoskeletal tumors, we hypothesized that regulation of mitochondrial biogenesis could be a potent therapeutic target for osteosarcoma.
Recent studies have highlighted the relationship between cancer cell growth and cell metabolism, and the energyregulating enzyme AMPK is thought to be a key kinase that 
mediates a metabolic cell cycle checkpoint (25). In human skeletal muscle, activation of AMPK functions to maintain cellular energy stores and to switch on catabolic pathways that produce ATP, mostly by enhancing oxidative metabolism and mitochondrial biogenesis, while switching off anabolic pathways that consume ATP; AMPK also directly activates PGC- $1 \alpha$ and regulates mitochondrial biogenesis (19). In tumor cells, the activation of AMPK results in tumor suppression through cell cycle arrest or apoptosis following phosphorylation of p53 and FOXO3a $(26,27)$ and induction of the cyclin-dependent kinase inhibitors $\mathrm{p} 21^{\mathrm{cip} 1}$ and $\mathrm{p} 27^{\mathrm{kip} 1}$, leading to cell cycle arrest $(26,28)$. In this study, we examined the therapeutic potential of mitochondrial proliferation via PGC-1 $\alpha$ activation by AMPK in human osteosarcoma cells.

In order to activate the AMPK/PGC- $1 \alpha /$ TFAM axis in mitochondrial biogenesis to fight cancer by interfering with energy metabolism in cancer cells, AMPK activators, such as the guanidine derivative metformin or AICAR, have been used (29-33). AICAR is a nucleoside analog, which initially was developed as a cardioprotective agent, but has a different mechanism of action than standard nucleoside analogs such as fludarabine (34). In the 1980s and 1990s, AICAR was under development as an agent that would reduce cardiac injury associated with coronary artery bypass grafting. Although initial trials clearly demonstrated success, later trials showed a minimal efficacy as the morbidity and mortality rates associated with the procedure decreased dramatically owing to better techniques and increased familiarity with the procedures $(35,36)$. When added to cultured cells or administered into animals or humans, AICAR is phosphorylated to become AICA-ribotide (ZMP), the natural endogenous intermediate in de novo purine nucleotide biosynthesis, which can function as an AMP mimic and can activate AMPK. Previous studies have shown that AICAR has anticancer effects in cancer cells $(18,30,32,37)$. For example, in HeLa cervical carcinoma cells, AMPK phosphorylation by AICAR increases the levels of $P G C-1 \alpha, N R F-1$ and TFAM mRNAs, resulting in upregulation of mtDNA replication and transcription (37). Additionally, other studies have revealed that AICAR inhibits cell proliferation and induces apoptosis in various cancer cells, including neuroblastoma (30), colon cancer (32), breast cancer, and prostate cancer cells (18). However, in osteosarcoma, the potential role of AMPK and/or the anticancer effects of AICAR through mitochondrial proliferation have not been addressed. In the present study, we hypothesized that AICAR could induce mitochondrial apoptosis in human osteosarcoma by increasing PGC-1 $\alpha$ expression through AMPK activation. In in vitro studies, we proved that AICAR phosphorylated AMPK and increased the expression of both PGC-1 $\alpha$ and TFAM, resulting in mitochondrial proliferation. Additionally, we demonstrated that the apoptotic activity was increased along with mitochondrial proliferation in AICAR-treated osteosarcoma cells. Furthermore, we showed that AICAR suppressed in vivo osteosarcoma cell growth by inducing mitochondrial apoptosis without apparent body weight loss. Our results strongly suggested that decreased mitochondrial numbers contributed to the progression of human osteosarcoma, and that improvement of mitochondrial biogenesis by AICAR through AMPK activation had anticancer effects on human osteosarcoma via induction of mitochondrial proliferation and apoptosis.

In the present study, we employed AICAR as an AMPK activator for all experiments. Another AMPK activator, metformin, which is typically the first drug used to treat patients with diabetes mellitus type 2 (38), activates AMPK by affecting the mitochondrial complex I of the respiratory chain, leading to a drop in intracellular ATP levels (39). Thus, metformin may inhibit the function of isolated mitochondria (40-43), while AICAR has been reported to activate mitochondrial function in skeletal muscle via direct phosphorylation of PGC-1 $\alpha$ (19). Additionally, metformin can induce various side effects, including onset of lactic acidosis. Therefore, in the clinical setting, metformin may not be feasible to use in patients without diabetes mellitus. In contrast, AICAR is relatively safe, as shown in clinical trials (36). Additionally, compared with standard chemotherapies, AICAR exhibits preferential toxicity for tumor cells and does not cause substantial damage to non-tumor cells (44).

In conclusion, we demonstrated that AICAR induced mitochondrial apoptosis via the PGC- $1 \alpha / \mathrm{TFAM} /$ mitochondrial pathway by AMPK phosphorylation in human osteosarcoma. To the best of our knowledge, this is the first study describing the apoptotic effects of AICAR through the AMPK/PGC-1 $\alpha /$ TFAM axis in the mitochondrial pathway in human osteosarcoma. However, this study had several limitations. We found that AICAR could decrease in vivo osteosarcoma tumor growth but did not reduce tumor size; unfortunately, we did not assess the antitumor effects of AICAR in the context of AMPK inhibition by siRNA or AMPK inhibitors, such as compound $\mathrm{C}(30,31)$. Although further studies are needed to determine whether AICAR has sufficient effects in human osteosarcoma and to elucidate the mechanisms mediating the antitumor effects of AICAR, mitochondrial biogenesis via the AMPK/PGC-1 $\alpha /$ TFAM pathway should be an attractive target for osteosarcoma, and AICAR may be a potent therapeutic agent for the treatment of osteosarcoma.

\section{Acknowledgements}

We thank Minako Nagata, Maya Yasuda, and Kyoko Tanaka for their expert technical assistance.

\section{References}

1. Hegyi M, Semsei AF, Jakab Z, Antal I, Kiss J, Szendroi M, Csoka M and Kovacs G: Good prognosis of localized osteosarcoma in young patients treated with limb-salvage surgery and chemotherapy. Pediatr Blood Cancer 57: 415-422, 2011.

2. Jaffe N: Osteosarcoma: Review of the past, impact on the future. The American experience. Cancer Treat Res 152: 239-262, 2009.

3. van Maldegem AM, Bhosale A, Gelderblom HJ, Hogendoorn PC and Hassan AB: Comprehensive analysis of published phase I/II clinical trials between 1990-2010 in osteosarcoma and Ewing sarcoma confirms limited outcomes and need for translational investment. Clin Sarcoma Res 2: 5, 2012.

4. Chan DC: Mitochondria: Dynamic organelles in disease, aging, and development. Cell 125: 1241-1252, 2006.

5. Neuzil J, Dong LF, Rohlena J, Truksa J and Ralph SJ: Classification of mitocans, anti-cancer drugs acting on mitochondria. Mitochondrion 13: 199-208, 2013.

6. Yamada S, Nomoto S, Fujii T, Kaneko T, Takeda S, Inoue S, Kanazumi $\mathrm{N}$ and Nakao A: Correlation between copy number of mitochondrial DNA and clinico-pathologic parameters of hepatocellular carcinoma. Eur J Surg Oncol 32: 303-307, 2006. 
7. Yu M,Zhou Y, Shi Y, Ning L, Yang Y, Wei X,Zhang N,Hao X and Niu R: Reduced mitochondrial DNA copy number is correlated with tumor progression and prognosis in Chinese breast cancer patients. IUBMB Life 59: 450-457, 2007.

8. Wu CW, Yin PH, Hung WY, Li AF, Li SH, Chi CW, Wei YH and Lee HC: Mitochondrial DNA mutations and mitochondrial DNA depletion in gastric cancer. Genes Chromosomes Cancer 44: $19-28,2005$.

9. Lee HC, Li SH, Lin JC, Wu CC, Yeh DC and Wei YH: Somatic mutations in the D-loop and decrease in the copy number of mitochondrial DNA in human hepatocellular carcinoma. Mutat Res 547: 71-78, 2004.

10. Xing J, Chen M, Wood CG, Lin J, Spitz MR, Ma J, Amos CI, Shields PG, Benowitz NL, Gu J, et al: Mitochondrial DNA content: Its genetic heritability and association with renal cell carcinoma. J Natl Cancer Inst 100: 1104-1112, 2008.

11. Tseng LM, Yin PH, Chi CW, Hsu CY, Wu CW, Lee LM, Wei YH and Lee HC: Mitochondrial DNA mutations and mitochondrial DNA depletion in breast cancer. Genes Chromosomes Cancer 45: 629-638, 2006

12. Kim MM, Clinger JD, Masayesva BG, Ha PK, Zahurak ML, Westra WH and Califano JA: Mitochondrial DNA quantity increases with histopathologic grade in premalignant and malignant head and neck lesions. Clin Cancer Res 10: 8512-8515, 2004.

13. Lin CS, Chang SC, Wang LS, Chou TY, Hsu WH, Wu YC and Wei YH: The role of mitochondrial DNA alterations in esophageal squamous cell carcinomas. J Thorac Cardiovasc Surg 139: 189-197.e4, 2010

14. Wang Y, Liu VW, Xue WC, Cheung AN and Ngan HY: Association of decreased mitochondrial DNA content with ovarian cancer progression. Br J Cancer 95: 1087-1091, 2006.

15. Onishi Y, Ueha T, Kawamoto T, Hara H, Toda M, Harada R, Minoda M, Kurosaka M and Akisue T: Regulation of mitochondrial proliferation by PGC-1 $\alpha$ induces cellular apoptosis in musculoskeletal malignancies. Sci Rep 4: 3916, 2014.

16. Ekstrand MI, Falkenberg M, Rantanen A, Park CB, Gaspari M, Hultenby K, Rustin P, Gustafsson CM and Larsson NG: Mitochondrial transcription factor A regulates mtDNA copy number in mammals. Hum Mol Genet 13: 935-944, 2004

17. Corton JM, Gillespie JG, Hawley SA and Hardie DG: 5-Aminoimidazole-4-carboxamide ribonucleoside. A specific method for activating AMP-activated protein kinase in intact cells? Eur J Biochem 229: 558-565, 1995.

18. Swinnen JV, Beckers A, Brusselmans K, Organe S, Segers J, Timmermans L, Vanderhoydonc F, Deboel L, Derua R, Waelkens E, et al: Mimicry of a cellular low energy status blocks tumor cell anabolism and suppresses the malignant phenotype. Cancer Res 65: 2441-2448, 2005.

19. Jäger S, Handschin C, St-Pierre J and Spiegelman BM: AMP-activated protein kinase (AMPK) action in skeletal muscle via direct phosphorylation of PGC-1alpha. Proc Natl Acad Sci USA 104: 12017-12022, 2007.

20. Narkar VA, Downes M, Yu RT, Embler E, Wang YX, Banayo E, Mihaylova MM, Nelson MC, Zou Y, Juguilon H, et al: AMPK and PPARdelta agonists are exercise mimetics. Cell 134: 405-415, 2008

21. Okada Y, Akisue T, Hara H, Kishimoto K, Kawamoto T, Imabori M, Kishimoto S, Fukase N, Onishi Y and Kurosaka M: The effect of bevacizumab on tumour growth of malignant fibrous histiocytoma in an animal model. Anticancer Res 30: 3391-3395, 2010

22. Yu M, Wan Y and Zou Q: Decreased copy number of mitochondrial DNA in Ewing's sarcoma. Clin Chim Acta 411: 679-683, 2010

23. Friebele JC, Peck J, Pan X, Abdel-Rasoul M and Mayerson JL: Osteosarcoma: A Meta-Analysis and Review of the Literature. Am J Orthop (Belle Mead NJ) 44: 547-553, 2015.

24. Mirabello L, Troisi RJ and Savage SA: Osteosarcoma incidence and survival rates from 1973 to 2004: Data from the Surveillance, Epidemiology, and End Results Program. Cancer 115: 1531-1543, 2009.

25. Sanli T, Rashid A, Liu C, Harding S, Bristow RG, Cutz JC, Singh $\mathrm{G}$, Wright $\mathrm{J}$ and Tsakiridis T: Ionizing radiation activates AMP-activated kinase (AMPK): A target for radiosensitization of human cancer cells. Int J Radiat Oncol Biol Phys 78: 221-229, 2010.

26. Gwinn DM, Shackelford DB, Egan DF, Mihaylova MM, Mery A, Vasquez DS, Turk BE and Shaw RJ: AMPK phosphorylation of raptor mediates a metabolic checkpoint. Mol Cell 30: 214-226, 2008 .
27. Shackelford DB and Shaw RJ: The LKB1-AMPK pathway: Metabolism and growth control in tumour suppression. Nat Rev Cancer 9: 563-575, 2009.

28. Mihaylova MM and Shaw RJ: The AMPK signalling pathway coordinates cell growth, autophagy and metabolism. Nat Cell Biol 13: 1016-1023, 2011.

29. Cheng J, Huang T, Li Y, Guo Y, Zhu Y, Wang Q, Tan X, Chen W, Zhang $\mathrm{Y}$, Cheng $\mathrm{W}$, et al: AMP-activated protein kinase suppresses the in vitro and in vivo proliferation of hepatocellular carcinoma. PLoS One 9: e93256, 2014.

30. Guo D, Hildebrandt IJ, Prins RM, Soto H, Mazzotta MM, Dang J, Czernin J, Shyy JY, Watson AD, Phelps M, et al: The AMPK agonist AICAR inhibits the growth of EGFRvIII-expressing glioblastomas by inhibiting lipogenesis. Proc Natl Acad Sci USA 106: 12932-12937, 2009

31. Sauer H, Engel S, Milosevic N, Sharifpanah F and Wartenberg M: Activation of AMP-kinase by AICAR induces apoptosis of DU-145 prostate cancer cells through generation of reactive oxygen species and activation of c-Jun N-terminal kinase. Int J Oncol 40: 501-508, 2012.

32. Su RY, Chao Y, Chen TY, Huang DY and Lin WW: 5-Aminoimidazole-4-carboxamide riboside sensitizes TRAIL-and TNF alpha\}-induced cytotoxicity in colon cancer cells through AMP-activated protein kinase signaling. Mol Cancer Ther 6: 1562-1571, 2007

33. Woodard J and Platanias LC: AMP-activated kinase (AMPK)generated signals in malignant melanoma cell growth and survival. Biochem Biophys Res Commun 398: 135-139, 2010.

34. Van Den Neste E, Cazin B, Janssens A, González-Barca E, Terol MJ, Levy V, Pérez de Oteyza J, Zachee P, Saunders A, de Frias M, et al: Acadesine for patients with relapsed/refractory chronic lymphocytic leukemia (CLL): A multicenter phase I/II study. Cancer Chemother Pharmacol 71: 581-591, 2013.

35. Aronson S: An initial multicenter, randomized controlled trial on the safety and efficacy of acadesine in patients undergoing coronary artery bypass graft surgery. Anesth Analg 79: 1021-1022, 1994.

36. Newman MF, Ferguson TB, White JA, Ambrosio G, Koglin J, Nussmeier NA, Pearl RG, Pitt B, Wechsler AS, Weisel RD, et al; RED-CABG Steering Committee and Investigators: Effect of adenosine-regulating agent acadesine on morbidity and mortality associated with coronary artery bypass grafting: The RED-CABG randomized controlled trial. JAMA 308: 157-164, 2012.

37. Fu X, Wan S, Lyu YL, Liu LF and Qi H: Etoposide induces ATM-dependent mitochondrial biogenesis through AMPK activation. PLoS One 3: e2009, 2008.

38. Zhou G, Myers R, Li Y, Chen Y, Shen X, Fenyk-Melody J, Wu M, Ventre J, Doebber T, Fujii N, et al: Role of AMP-activated protein kinase in mechanism of metformin action. J Clin Invest 108: 1167-1174, 2001.

39. Hawley SA, Ross FA, Chevtzoff C, Green KA, Evans A, Fogarty S, Towler MC, Brown LJ, Ogunbayo OA, Evans AM, et al: Use of cells expressing gamma subunit variants to identify diverse mechanisms of AMPK activation. Cell Metab 11: 554-565, 2010

40. El-Mir MY, Nogueira V, Fontaine E, Avéret N, Rigoulet M and Leverve X: Dimethylbiguanide inhibits cell respiration via an indirect effect targeted on the respiratory chain complex I. J Biol Chem 275: 223-228, 2000.

41. Owen MR, Doran E and Halestrap AP: Evidence that metformin exerts its anti-diabetic effects through inhibition of complex 1 of the mitochondrial respiratory chain. Biochem J 348: 607-614, 2000 .

42. Brunmair B, Staniek K, Gras F, Scharf N, Althaym A, Clara R, Roden M, Gnaiger E, Nohl H, Waldhäusl W, et al: Thiazolidinediones, like metformin, inhibit respiratory complex I: A common mechanism contributing to their antidiabetic actions? Diabetes 53: 1052-1059, 2004.

43. Turner N, Li JY, Gosby A, To SW, Cheng Z, Miyoshi H, Taketo MM, Cooney GJ, Kraegen EW, James DE, et al: Berberine and its more biologically available derivative, dihydroberberine, inhibit mitochondrial respiratory complex I: A mechanism for the action of berberine to activate AMP-activated protein kinase and improve insulin action. Diabetes 57: 1414-1418, 2008.

44. Jose C, Hébert-Chatelain E, Bellance N, Larendra A, Su M, Nouette-Gaulain K and Rossignol R: AICAR inhibits cancer cell growth and triggers cell-type distinct effects on OXPHOS biogenesis, oxidative stress and Akt activation. Biochim Biophys Acta 1807: 707-718, 2011. 